\title{
UNIVALENT FUNCTIONS WITH NEGATIVE COEFFICIENTS
}

\author{
HERB SILVERMAN
}

ABSTRACT. Coefficient, distortion, covering, and coefficient inequalities are determined for univalent functions with negative coefficients that are starlike of order $a$ and convex of order $a_{0}$ Extreme points for these classes are also determined.

1. Introduction. Let $S$ denote the class of functions of the form $f(z)=$ $z+\sum_{n=2}^{\infty} a_{n} z^{n}$ that are analytic and univalent in the unit disk $|z|<1$. A function $f \in S$ is said to be starlike of order $\alpha(0 \leq \alpha<1)$, denoted $f \in S^{*}(\alpha)$, if $\operatorname{Re}\left\{z f^{\prime} / f\right\}>a(|z|<1)$ and is said to be convex of order $a$, denoted $f \in$ $K(\alpha)$, if $\operatorname{Re}\left\{1+z f^{\prime \prime} / f^{\prime}\right\}>a(|z|<1)$.

Let $T$ denote the subclass of $S$ consisting of functions whose nonzero coefficients, from the second on, are negative. That is, an analytic and univalent function $f$ is in $T$ if it can be expressed as

$$
f(z)=z-\sum_{n=2}^{\infty}\left|a_{n}\right| z^{n} .
$$

We also denote by $T^{*}(\alpha)$ and $C(\alpha)$ the subclasses of $T$ that are, respectively, starlike of order $\alpha$ and convex of order $\alpha$.

In [5], Schild investigated the subclass of $T$ consisting of polynomials. We show that the coefficient bounds, distortion, covering, and radius of convexity theorems he obtained remain valid for the whole class $T$. We also prove comparable theorems for the classes $T^{*}(\alpha)$ and $C(\alpha)$. In addition, we show that a function in $C(\alpha)$ must be in $T^{*}(2 /(3-\alpha))$. In the sequel, we shall assume that the coefficients of a function in $T$ are defined by (1).

2. Coefficient inequalities. We begin with a theorem that relates the modulus of the coefficients to the order of starlikeness.

Presented to the Society, January 24, 1975; received by the editors May 16, 1974. $30 \mathrm{~A} 40$.

AMS (MOS) subject classifications (1970). Primary 30A32; Secondary 30A34,

Key words and phrases. Univalent functions, starlike, convex, radius of convexity, extreme points. 
Theorem 1. Let $f(z)=z+\sum_{n=2}^{\infty} a_{n} z^{n}$. If $\sum_{n=2}^{\infty}(n-\alpha)\left|a_{n}\right| \leq 1-\alpha$, then $f \in S^{*}(\alpha)$.

Proof. It suffices to show that the values for $z f^{\prime} / f$ lie in a circle centered at $w=1$ whose radius is $1-\alpha$. We have

$$
\begin{aligned}
\left|z \frac{f^{\prime}}{f}-1\right| & =\left|\frac{z f^{\prime}-f}{f}\right|=\left|\frac{\sum_{n=2}^{\infty}(n-1) a_{n} z^{n}}{z+\sum_{n=2}^{\infty} a_{n} z^{n}}\right| \\
& \leq \frac{\sum_{n=2}^{\infty}(n-1)\left|a_{n}\right||z|^{n-1}}{1-\sum_{n=2}^{\infty}\left|a_{n}\right||z|^{n-1}} \leq \frac{\sum_{n=2}^{\infty}(n-1)\left|a_{n}\right|}{1-\sum_{n=2}^{\infty}\left|a_{n}\right|} .
\end{aligned}
$$

This last expression is bounded above by $1-\alpha$ if

$$
\sum_{n=2}^{\infty}(n-1)\left|a_{n}\right| \leq(1-\alpha)\left(1-\sum_{n=2}^{\infty}\left|a_{n}\right|\right)
$$

which is equivalent to

$$
\sum_{n=2}^{\infty}(n-a)\left|a_{n}\right| \leq 1-a .
$$

But (2) is true by hypothesis. Hence $\left|z f^{\prime} / f-1\right| \leq 1-\alpha$, and the theorem is proved.

Special cases of Theorem 1 have been proved by Goodman [2], $\alpha=0$, and by Schild [6], $\alpha=1 / 2$.

Corollary. Let $f(z)=z+\sum_{n=2}^{\infty} a_{n} z^{n}$. If $\sum_{n=2}^{\infty} n(n-\alpha)\left|a_{n}\right| \leq 1-\alpha$, then $f \in K(\alpha)$.

Proof. It is well known that $f \in K(\alpha)$ if and only if $z f^{\prime} \in S^{*}(\alpha)$. Since $z f^{\prime}=z+\sum_{n=2}^{\infty} n a_{n} z^{n}$, we may replace $a_{n}$ with $n a_{n}$ in the theorem.

For functions in $T^{*}(\alpha)$, the converse of Theorem 1 is also true.

Theorem 2. A function $f(z)=z-\sum_{n=2}^{\infty}\left|a_{n}\right| z^{n}$ is in $T^{*}(\alpha)$ if and only if $\sum_{n=2}^{\infty}(n-a)\left|a_{n}\right| \leq 1-a$.

Proof. In view of Theorem 1, it suffices to show the only if part. Assume that

$$
\operatorname{Re}\left\{z \frac{f^{\prime}}{f}\right\}=\operatorname{Re}\left\{\frac{z-\sum_{n=2}^{\infty} n\left|a_{n}\right| z^{n}}{z-\sum_{n=2}^{\infty}\left|a_{n}\right| z^{n}}\right\}>a \quad(|z|<1)
$$


Choose values of $z$ on the real axis so that $z f^{\prime} / f$ is real. Upon clearing the denominator in (3) and letting $z \rightarrow 1$ through real values, we obtain

$$
1-\sum_{n=2}^{\infty} n\left|a_{n}\right| \geq a\left(1-\sum_{n=2}^{\infty}\left|a_{n}\right|\right)
$$

Thus $\sum_{n=2}^{\infty}(n-\alpha)\left|a_{n}\right| \leq 1-\alpha$, and the proof is complete.

Corollary 1. If $f \in T^{*}(\alpha)$ then $\left|a_{n}\right| \leq(1-\alpha) /(n-\alpha)$, with equality only for functions of the form $f_{n}(z)=z-(1-\alpha) z^{n} /(n-\alpha)$.

Corollary 2. A function $f(z)=z-\sum_{n=2}^{\infty}\left|a_{n}\right| z^{n}$ is in $C(\alpha)$ if and only if $\sum_{n=2}^{\infty} n(n-\alpha)\left|a_{n}\right| \leq 1-\alpha$.

Proof. The proof follows as that of the corollary to Theorem 1.

Theorem 3. If $f \in T$, then $\sum_{n=2}^{\infty} n\left|a_{n}\right| \leq 1$.

Proof. Suppose $\sum_{n=2}^{\infty} n\left|a_{n}\right|=1+\epsilon(\epsilon>0)$. Then there exists an integer $N$ such that $\sum_{n=2}^{N} n\left|a_{n}\right|>1+\epsilon / 2$. For $z$ in the interval $(1 /(1+\epsilon / 2))^{1 /(N-1)}$ $<z<1$, we have

$$
f^{\prime}(z) \leq 1-\sum_{n=2}^{N} n\left|a_{n}\right| z^{n-1} \leq 1-z^{N-1} \sum_{n=2}^{N} n\left|a_{n}\right|<1-\left(1+\frac{\epsilon}{2}\right) z^{N-1}<0 .
$$

Since $f^{\prime}(0)>0$, there exists a real number $z_{0}, 0<z_{0}<1$, for which $f^{\prime}\left(z_{0}\right)$ $=0$. Hence $f \notin T$.

Corollary. $T=T^{*}(0)$.

Proof. Set $a=0$ in Theorem 2.

3. Distortion and covering theorems for $T^{*}(\alpha)$ and $C(\alpha)$. Our coefficient bounds enable us to prove

Theorem 4. If $f \in T^{*}(\alpha)$, then

$$
r-\frac{1-\alpha}{2-\alpha} r^{2} \leq|f| \leq r+\frac{1-\alpha}{2-\alpha} r^{2} \quad(|z|=r),
$$

with equality for $f(z)=z-(1-\alpha) z^{2} /(2-\alpha)(z= \pm r)$.

Proof. Note that $(2-\alpha) \sum_{n=2}^{\infty}\left|a_{n}\right| \leq \sum_{n=2}^{\infty}(n-\alpha)\left|a_{n}\right| \leq 1-\alpha$, this last inequality following from Theorem 2 . Thus

$$
|f(z)| \leq r+\sum_{n=2}^{\infty}\left|a_{n}\right| r^{n} \leq r+r^{2} \sum_{n=2}^{\infty}\left|a_{n}\right| \leq r+\frac{1-\alpha}{2-a} r^{2} .
$$


Similarly,

$$
|f(z)| \geq r-\sum_{n=2}^{\infty}\left|a_{n}\right| r^{n} \geq r-r^{2} \sum_{n=2}^{\infty}\left|a_{n}\right| \geq r-\frac{1-\alpha}{2-\alpha} r^{2}
$$

Corollary. If $f \in C(\alpha)$, then

$$
r-\frac{1-\alpha}{2(2-\alpha)} r^{2} \leq|f| \leq r+\frac{1-\alpha}{2(2-\alpha)} r^{2} \quad(|z|=r)
$$

with equality for $f(z)=z-(1-\alpha) z^{2} / 2(2-\alpha)(z= \pm r)$.

Theorem 5. The disk $|z|<1$ is mapped onto a domain that contains the disk $|w|<1 /(2-\alpha)$ by any $f \in T^{*}(\alpha)$, and onto a domain that contains the disk $|w|<(3-\alpha) /(4-2 \alpha)$ by any $f \in C(\alpha)$. The theorem is sharp, with extremal functions $z-(1-\alpha) z^{2} /(2-\alpha) \in T^{*}(\alpha)$ and $z-(1-\alpha) z^{2} / 2(2-\alpha)$ $\epsilon(\alpha)$.

Proof. The results follow upon letting $r \rightarrow 1$ in Theorem 4 and its corollary.

Theorem 6. If $f \in T^{*}(\alpha)$, then

$$
1-\frac{2(1-\alpha)}{2-\alpha} r \leq\left|f^{\prime}\right| \leq 1+\frac{2(1-\alpha)}{2-\alpha} r \quad(|z|=r) .
$$

Equality holds for $f(z)=z-(1-\alpha) z^{2} /(2-\alpha)(z= \pm r)$.

Proof. We have

$$
\left|f^{\prime}\right| \leq 1+\sum_{n=2}^{\infty} n\left|a_{n}\right||z|^{n-1} \leq 1+r \sum_{n=2}^{\infty} n\left|a_{n}\right|
$$

In view of Theorem 2,

$$
\sum_{n=2}^{\infty} n\left|a_{n}\right| \leq 1-\alpha+\alpha \sum_{n=2}^{\infty}\left|a_{n}\right| \leq 1-\alpha+\frac{\alpha(1-\alpha)}{2-\alpha}=\frac{2(1-\alpha)}{2-\alpha}
$$

A substitution of (5) into (4) yields the right-hand inequality.

On the other hand,

$$
\left|f^{\prime}\right| \geq 1-\sum_{n=2}^{\infty} n\left|a_{n}\right||z|^{n-1} \geq 1-r \sum_{n=2}^{\infty} n\left|a_{n}\right| \geq 1-\frac{2(1-\alpha)}{2-\alpha} r .
$$

This completes the proof.

Corollary. If $f \in C(\alpha)$, then 


$$
1-\frac{1-a}{2-a} r \leq\left|f^{\prime}\right| \leq 1+\frac{1-a}{2-a} r \quad(|z|=r) .
$$

Equality holds for $f(z)=z-(1-\alpha) z^{2} / 2(2-\alpha)(z= \pm r)$.

4. Order of starlikeness and radius of convexity theorems for the classes $C(\alpha)$ and $T^{*}(\alpha)$. Recently MacGregor [4] has obtained sharp bounds on the order of starlikeness for functions in $K(\alpha)$. We now do this for functions in $C(\alpha)$.

Theorem 7. If $f \in C(\alpha)$, then $f \in T^{*}(2 /(3-\alpha))$. The result is sharp, with $f(z)=z-(1-\alpha) z^{2} / 2(2-\alpha)$ being extremal.

Proof, In view of Theorem 2 and Corollary 2, we must prove that

$$
\sum_{n=2}^{\infty} \frac{n(n-\alpha)}{1-\alpha}\left|a_{n}\right| \leq 1 \Rightarrow \sum_{n=2}^{\infty} \frac{n-2 /(3-\alpha)}{1-2 /(3-\alpha)}\left|a_{n}\right| \leq 1 .
$$

It suffices to show that

$$
\frac{n(n-\alpha)}{1-\alpha} \geq \frac{n-2 /(3-\alpha)}{1-2 /(3-\alpha)}=\frac{n(3-\alpha)-2}{1-\alpha} \quad(n=2,3, \ldots) .
$$

But (6) is equivalent to $n^{2}-3 n+2 \geq 0(n=2,3, \ldots)$, and the theorem is proved.

There is no converse to Theorem 7. That is, a function in $T^{*}(\alpha)$ need not be convex. To show this, we need only find coefficients $\left\{a_{n}\right\}$ for which

$$
\sum_{n=2}^{\infty} \frac{n-\alpha}{1-a}\left|a_{n}\right| \leq 1 \text { and } \sum_{n=2}^{\infty} n^{2}\left|a_{n}\right|>1 \text {. }
$$

Note that the functions $f_{n}(z)=z-(1-\alpha) z^{n} /(n-\alpha)(n>1 /(1-\alpha))$ all satisfy both inequalities in (7). We now determine the radius of convexity for functions in $T^{*}(\alpha)$.

Theorem 8. If $f \in T^{*}(\alpha)$, then $f$ is convex in the disk

$$
|z|<r=r(\alpha)=\inf _{n}\left(\frac{n-\alpha}{n^{2}(1-\alpha)}\right)^{1 /(n-1)} \quad(n=2,3, \cdots) .
$$

The result is sharp, with the extremal function being of the form $f_{n}(z)=z-$ $(1-\alpha) z^{n} /(n-\alpha)$ for some $n$.

Proof. It suffices to show that $\left|z f^{\prime \prime} / f^{\prime}\right| \leq 1$ for $|z| \leq r(\alpha)$. We have

$$
\left|z \frac{f^{\prime \prime}}{f^{\prime}}\right|=\left|\frac{-\sum_{n=2}^{\infty} n(n-1)\left|a_{n}\right| z^{n-1}}{1-\sum_{n=2}^{\infty} n\left|a_{n}\right| z^{n-1}}\right| \leq \frac{\sum_{n=2}^{\infty} n(n-1)\left|a_{n} \| z\right|^{n-1}}{1-\sum_{n=2}^{\infty} n\left|a_{n}\right||z|^{n-1}}
$$


Thus $\left|z f^{\prime \prime} / f^{\prime}\right| \leq 1$ if

or

$$
\sum_{n=2}^{\infty} n(n-1)\left|a _ { n } \left\|\left.z\right|^{n-1} \leq 1-\sum_{n=2}^{\infty} n\left|a_{n} \| z\right|^{n-1},\right.\right.
$$

$$
\sum_{n=2}^{\infty} n^{2}\left|a_{n}\right||z|^{n-1} \leq 1 .
$$

According to Theorem 2, $\sum_{n=2}^{\infty}(n-\alpha)\left|a_{n}\right| /(1-\alpha) \leq 1$. Hence (8) will be true if

$$
n^{2}|z|^{n-1} \leq(n-\alpha) /(1-\alpha) \quad(n=2,3, \cdots) .
$$

Solving (9) for $|z|$, we obtain

$$
|z| \leq\left((n-\alpha) / n^{2}(1-\alpha)\right)^{1 /(n-1)} \quad(n=2,3, \cdots) .
$$

Setting $|z|=r(\alpha)$ in (10), the result follows.

Remarks. The integer $n$ that yields the sharp result in Theorem 8 is a nondecreasing function of $\alpha$, and grows arbitrarily large as $\alpha$ tends to 1 . For $0 \leq \alpha \leq 2-4 \sqrt{ } 7 / 7=.487 \ldots, n=2$, so that $r(\alpha)=(2-\alpha) / 4(1-\alpha)$. For $2-4 \sqrt{ } 7 / 7 \leq \alpha \leq .572 \ldots, n=3$, so that $r(\alpha)=((3-\alpha) /(1-\alpha))^{1 / 2} / 3$. The radius of convexity for $S^{*}(0)$ is known to be $2-\sqrt{3}$. MacGregor [3] has shown that the radius of convexity for $S^{*}(1 / 2)$ is $\sqrt{2 \sqrt{3}-3}$. The general radius of convexity problem for $S^{*}(\alpha)$ appears to be open.

5. Extreme points for $T^{*}(\alpha)$ and $C(\alpha)$. For any compact family $F$ of univalent functions, the maximum or minimum value on $F$ of the real part of any continuous linear functional defined over the set of analytic functions occurs at one of the extreme points of the closed convex hull of $F$. Consequently, the determination of the extreme points of a family $F$ enables us to solve many extremal problems for $F$. To illustrate, the Bieberbach conjecture would be solved if the conjecture could be settled for the extreme points of $S$. In a landmark paper [1], Brickman, MacGregor, and Wilkin found the extreme points of the closed convex hull for convex, starlike, close-to-convex, and typically real functions. Since then, the extreme points for many additional classes have been determined.

In view of Theorem 2 , the class $T^{*}(\alpha)$ is closed under convex linear combinations. We shall now determine the extreme points of $T^{*}(\alpha)$.

Theorem 9. Let $f_{1}(z)=z$ and $f_{n}(z)=z-(1-\alpha) z^{n} /(n-\alpha)(n=2,3$, ...). Then $f \in T^{*}(\alpha)$ if and only if it can be expressed in the form $f(z)=$ $\sum_{n=1}^{\infty} \lambda_{n} f_{n}(z)$, where $\lambda_{n} \geq 0$ and $\sum_{n=1}^{\infty} \lambda_{n}=1$. 
Proof. Suppose $f(z)=\Sigma_{n=1}^{\infty} \lambda_{n} f_{n}(z)=z-\sum_{n=2}^{\infty} \lambda_{n}(1-\alpha) z^{n} /(n-\alpha)$. Then

$$
\sum_{n=2}^{\infty} \lambda_{n} \frac{1-\alpha}{n-\alpha}\left(\frac{n-\alpha}{1-\alpha}\right)=\sum_{n=2}^{\infty} \lambda_{n}=1-\lambda_{1} \leq 1 .
$$

Thus $f \in T^{*}(\alpha)$.

Conversely, suppose $f \in T^{*}(\alpha)$. Since $\left|a_{n}\right| \leq(1-\alpha) /(n-\alpha)(n=2,3$, $\ldots)$, we may set $\lambda_{n}=(n-\alpha)\left|a_{n}\right| /(1-\alpha)(n=2,3, \ldots)$, and $\lambda_{1}=1-$ $\sum_{n=2}^{\infty} \lambda_{n}$. Then $f(z)=\sum_{n=1}^{\infty} \lambda_{n} f_{n}(z)$. This completes the proof.

Corollary 1. The extreme points of $T^{*}(\alpha)$ are the functions $f_{n}(z)(n=$ $1,2, \ldots)$.

Corollary 2. The extreme points of $C(\alpha)$ are given by $f_{1}(z)=z$ and $f_{n}(z)=z-(1-\alpha) z^{n} / n(n-\alpha)(n=2,3, \ldots)$.

Proof. The operator $L$ defined by $L f(z)=\int_{0}^{z} f(t) / t d t$ is a linear homeomorphism from $T^{*}(\alpha)$ to $C(\alpha)$.

We are now furnished with an alternate method for proving some of our previous theorems. For example Theorem 4, Theorem 6, and their corollaries may be proved by examining the extreme points for the appropriate classes.

Most subclasses of $S$ are defined in terms of a geometric restriction on the image domain (starlike, convex, close-to-convex, etc.). We close with a problem concerning a subclass of $S$ defined in terms of a geometric restriction on the coefficients.

Open problem. Suppose $f(z)=z+e^{i \lambda} \Sigma_{n=2}^{\infty}\left|a_{n}\right| z^{n}$ is in $S(0 \leq|\lambda| \leq \pi)$. Can we find a sharp function $g(\lambda, n)$ for which $\left|a_{n}\right| \leq g(\lambda, n)$ for every $n$ ?

Here we have restricted the coefficients to a ray whose argument is $\lambda$. Note that $g(0, n)=n$, with the Koebe function $f(z)=z /(1-z)^{2}$ being extremal; and $g(\pi, n)=1 / n$ with extremal function $f(z)=z-z^{n} / n$.

Is $g(\lambda, n)$ a decreasing function of $|\lambda|$, perhaps something like $g(\lambda, n)$ $=n^{\cos \lambda}$ ? For $0<|\lambda|<\pi$, nothing is known. We do not even know if the Bieberbach conjecture is true.

\section{REFERENCES}

1. L. Brickman, T. H. MacGregor and D. R. Wilkin, Convex hulls of some classical families of univalent functions, Trans. Amer. Math. Soc. 156 (1971), 91-107. MR 43 \#494.

2. A. W. Goodman, Univalent functions and nonanalytic curves, Proc. Amer. Math. Soc. 8 (1957), 598-601. MR 19, 260. 
3. T. H. MacGregor, The radius of convexity for starlike functions of order $1 / 2$, Proc. Amer. Math. Soc. 14 (1963), 71-76. MR 27 \#283.

4. - - A subordination for convex functions of order a, J. London Math Soc. (to appear).

5. A. Schild, On a class of functions schlicht in the unit circle, Proc. Amer. Math. Soc. 5 (1954), 115-120. MR 15, 694.

6. - On a class of univalent star shaped mappings, Proc. Amer. Math. Soc. 9 (1958), 751-757. MR $20 \# 2452$.

DEPARTMENT OF MATHEMATICS, CLARK UNIVERSITY, WORCESTER, MASSACHU SETTS 01610

Current address: Department of Mathematics, University of Delaware, Newark, Delaware 19711 